[18] S. Kaczmarz und H. Steinhaus, Theorie der Orthogonalrcihen, Warszawa-Lwów 1935.

[19] S. Kakutani, Concrete representation of abstract $(M)$-spaces, Ann. of Math. 2 (42) (1941), p. 994-1024.

[20] J. L. Kelley, Banach spaces with the extension property, Trans. Amer. Math. Soc. 72 (1952), p. 323-326.

[21] H. Komatuzaki, Sur les projections dans cerlaines espaces du type (B), Pros. Imp. Acad. Tokyo 16 (1940), p. 274-279.

[22] - Une remarque sur les projections des certains espaces du type $(B)$, ibidem 17 (1941), p. $238-240$.

[23] C. Kuratowski, Topologie $I$, Warszawa 1958.

[24] J. Marcinkiewicz, Quelques théorèmes sur les séries orthogonales, Ann. Soc. Pol. Math. 17 (1938), p. 51-56.

[25] F. J. Murray, On complementary manifolds and projections in spaces $L_{p}$ nd $l_{p}$, Trans. Amer. Math. Soc. 41 (1937), p. 138-152.

[26] L. Nachbin, A theorem of Hahn-Banach type for linear transformations, ibidem 68 (1950), p. $28-46$.

[27] В. Н. Н и ко ль с к и й, Наилучиая аппроксижачия и базис в пространстве Фреше, Докл. Акад. Наук СССР (Н. С.) 59 (1948), p. 639-642.

[28] A. Pełezyński, On the isomorphism of the spaces $m$ and $M$, M. Bull. Acad. Pol. Sei., Série math. astr. et phys. 6 (1958), p. 695-696.

[29] R. S. Philips, On linear transformations, Trans. Amer. Math. Soc. 48 (1940), p. $516-541$.

[30] A. Sobezyk, Projections in Minkowski and Banach spaces, Duke Math. J. 8 (1941), p. $78-106$.

[31] - Projections of the space $(m)$ on its subspace $\left(c_{0}\right)$, Bull. Amer. Math. Soc. 47 (1941), p. $938-947$.

[32] В. Ф. Гапошкин, Об одном свойстве безусловных базисов в пространстве $L^{p}$, Усп. мат. наук, вып. 4 (88), т. XIV (1959), p. 143-148.

[33] B. Grünbaum, Projections onto some function spaces, Technical Report 23, University of Kansas 1959, p. 1.14.

Reģu par la Rédaction le 5. 10.1959

\title{
On the theory of non-linear operator equations on conjugately similar spaces
}

by

S. YAMAMURO (Sapporo)

1. Introduction. It is the purpose of this paper to consider an eigenvalue problem for some operators $F$ which map a Banach space $R$ into the conjugate space $\bar{R}$. For this purpose, we take, as the Banach space $R$, a special kind of vector lattice, a conjugately similar space which has been introduced by Nakano [9]. Roughly speaking, this is a Banach space $R$ such that a one-to-one correspondence $T$ exists between $R$ and $\bar{R}$. This correspondence $T$ enables us to define a proper value $\lambda$ and a proper element $a \in R$ of the operator $F$ from $R$ into $\bar{R}$ by the following equation:

$$
F a=\lambda T a .
$$

In the case of $L_{p}$-spaces $(p>1)$, this definition agrees with that of E. S. Citlanadze [4].

The definitions and elementary properties of the conjugately similar spaces will be given in $\S 2$. In the next section we will prove a theorem of L. A. Ljusternik in its special form. The simple proof may be interesting. In $\S 4$ we will consider the eigenvalue problem of a non-linear operator. The last section contains an application.

We express here our hearty thanks to Dr. Musielak for his valuable remarks on various points in this paper.

2. Conjugately similar spaces. Let $R$ be a vector lattice which satisfies the following condition: for any system of positive elements $x_{1}$ $(\lambda \in \Lambda)$ there exists an "infimum" element $\bigcap_{\lambda_{\in A}} x_{\lambda}$. The conjugate space $\bar{R}$ of $R$ is the totality of all linear (additive and homogeneous) functionals $\bar{x}$ on $R$ which satisfy the following condition: if $x_{\lambda \downarrow \lambda \in \Lambda} 0\left({ }^{1}\right)$, then

$$
\inf _{\lambda \in \Lambda}\left|\bar{x}\left(x_{\lambda}\right)\right|=0 .
$$

(1) We write $x_{\lambda \downarrow \lambda \in \Lambda} 0$ when $\left\{x_{\lambda}(\lambda \in \Lambda)\right\}$ is a non-increasing directed system and $\bigcap_{\lambda}=0$. 
By this notion of conjugate space we define the reflexivity of $R$ ([9], p. 93).

Definition ([9], p. 258). $R$ is said to be conjugately similar if $R$ is reflexive and there exists a one-to-one correspondence $T$ between $R$ and $\bar{R}: R \ni x \leftrightarrow T x \in \bar{R}$ such that

(1) $T(-x)=-T x$

(2) $T x \geqslant T y$ if and only if $x \geqslant y$;

(3) $(T x, x)=0\left({ }^{2}\right), x \geqslant 0$ implies $x=0$.

The correspondence $T$ is called the conjugately similar correspondence.

By this correspondence $T$ we define a functional $m(x)$ on $R$ by

$$
m(x)=\int_{0}^{1}(T \xi x, x) d \xi .
$$

We have $m(x)<+\infty$ for any $x \in R$, because $m(x) \leqslant(T x, x)$. The functional $m(x)$ satisfies the following conditions:
1) $0 \leqslant m(x)<+\infty$;
2) $m(x)=0$ implies $x=0$;
3) $|x| \geqslant|y|$ implies $m(x) \geqslant m(y)$;
4) $x \cap y=0\left({ }^{3}\right)$ implies $m(x+y)=m(x)+m(y)$;
5) $m(\alpha x+\beta y) \leqslant \alpha m(x)+\beta m(y)$ if $\alpha+\beta=1, \alpha, \beta \geqslant 0$
6) $0 \leqslant x_{\lambda \uparrow_{\text {A A }} x}$ implies $\sup m\left(x_{\lambda}\right)=m(x)$ (see [9], p. 261).

Therefore, the space $R$ is a modulared semi-ordered linear space in the sense of $(9)\left({ }^{4}\right)$.

If we define $\bar{m}(\bar{x})$ for $\bar{x} \epsilon \bar{R}$ by the relation

$$
\bar{m}(\bar{x})=\sup _{x_{G} R}\{\bar{x}(x)-m(x)\},
$$

then $\bar{R}$ is also a modulared semi-ordered linear space. Moreover, we have

$$
\bar{m}(\bar{x})=\int_{0}^{1}\left(\bar{x}, T^{-1} \xi \bar{x}\right) d \xi
$$

and

$$
(T x, x)=m(x)+\bar{m}(T x) .
$$

(2) For $x \in R$ and $\bar{x} \in \bar{R},(\bar{x}, x)$ moans the value of $\bar{x}$ at $x$, namely, $\bar{x}(x)$

$\left(^{(3)}\right.$ Elements $x$ and $y$ are said to be mutually orthogonal if $|x| \cap|y|=0$.

(4) In [9] the modulared semi-orderud linear space has been defined by more general conditions. Namely, the conjugately similar space is a special class of modalared semi-ordered linear spaces.
By the modular, we define two kinds of norms:

$$
\|x\|=\inf _{\xi>0} \frac{1+m(\xi x)}{\xi} ; \quad\|x\| \|=\inf _{m(\xi x) \leqslant 1} \frac{1}{|\xi|},
$$

which satisfy the following relation:

$$
\||x|\| \leqslant\|x\| \leqslant 2|| \mid x\|\|,
$$

and $m(x)=1$ if and only if $\||x|\|=1$ (see [9], p. 179 and [12]). By these norms, $R$ is monotone complete $\left(^{5}\right)$, and, therefore, $R$ is a Banach space and the Banach dual of $R$ is $\bar{R}$ (see [1] and [13]).

Finally, we must illustrate the notion of projection operators, which are indispensable in this paper. A subset $N$ of $R$ is said to be normal if every element $x \in R$ can be decomposed into two orthogonal elements $y$ and $z$ such that

$$
x=y+z, \quad y \in N \quad \text { and } \quad z \in N^{\perp} \text {. }
$$

In this case, we define the projection operator $[N]$ by

$$
y=[N] x \quad(x \in R) .
$$

This operator $[N]$ is linear and idempotent $([9], \S 5)$. The set

$$
\{p\}^{\perp}=\{x \in R:|p| \cap|x|=0\}
$$

is normal for any element $p \in R$. We denote $\left[\{p\}^{\perp \perp}\right]$ by $[p]([9], \S 6)$.

Example 1. $L_{p}$ is the totality of all measurable functions $x(t)$ $(0 \leqslant t \leqslant 1)$ such that $\int_{0}^{1}|x(t)|^{p} d t<+\infty$. If $1<p<+\infty$, the conjugate space of $L_{p}$ is $L_{q}$, where $q=p /(p-1)$. If we put

$$
T x(t)=|x(t)|^{p-1} \operatorname{sign} x(t),
$$

$T$ is a conjugately similar correspondence between $L_{p}$ and $L_{q}$, and

$$
\begin{gathered}
m(x)=\frac{1}{p} \int_{0}^{1}|x(t)|^{p} d t \\
\|\mid x\|=\left(\frac{1}{p} \int_{0}^{1}|x(t)|^{p} d t\right)^{1 / p}, \quad\|x\|=p^{1 / p} q^{1 / \alpha}\|x\| .
\end{gathered}
$$

(5) $R$ is said to be monotone complete if $0 \leqslant x_{\lambda \uparrow \Lambda}$ and $\sup _{\lambda \in \Lambda} m\left(x_{\lambda}\right)<+\infty$ imply that $\left\{x_{\lambda}(\lambda \in \Lambda)\right\}$ is order-bounded. 
Example 2. Let $L_{\oplus}$ be an Orlicz space. We assume that the function $\varphi(\xi)$ which appears in the integral

$$
\Phi(\xi)=\int_{0}^{\xi} \varphi(\eta) d \eta
$$

is strictly increasing and its strictly increasing inverse function $\psi(\xi)$ generates the conjugate function $\Psi(\xi)$ by the following relation:

$$
\Psi(\xi)=\int_{0}^{\xi} \psi(\eta) d \eta
$$

Then, $L_{\Psi}$ is the conjugate space of $L_{\Phi}$ and, if both spaces satisfy condition $\left(\Delta_{2}\right)$ for large arguments $\left({ }^{6}\right)$, then

$$
T x(t)=\varphi\left(x^{+}(t)\right)-\varphi\left(x^{-}(t)\right)
$$

is a conjugately similar correspondence between $L_{\Phi}$ and $L_{\Psi}$. In this case

$$
m(x)=\int_{0}^{1} \Phi(|x(t)|) d t .
$$

Example 3. For a measurable function $p(t) \geqslant 1(0 \leqslant t \leqslant 1)$, we define a function space $L_{p(t)}$ as the totality of all measurable functions $x(t)$ such that

$$
\int_{0}^{1} \frac{1}{p(t)}|\xi x(t)|^{p(t)} d t<+\infty \quad \text { for some } \xi>0 .
$$

When

$$
1<\inf _{0 \leqslant t \leqslant 1} p(t) \leqslant \sup _{0 \leqslant t \leqslant 1} p(t)<+\infty,
$$

the conjugate space of $L_{p(t)}$ is $L_{q(t)}$ where $q(t)=p(t) /(p(t)-1)$, and

$$
T x(t)=|x(t)|^{p(t)-1} \operatorname{sign} x(t)
$$

is a conjugately similar correspondence between $L_{p(t)}$ and $L_{q(t)}$. We have

$$
m(x)=\int_{0}^{1} \frac{1}{p(t)}|x(t)|^{p(t)} d t .
$$

(') Namely, we assume $m(x)<+\infty$ for every $x(t) \in I_{\Phi}$. For the condition $\left(\Delta_{2}\right)$, see [5].
In this paper $R$ is assumed to be conjugately similar by the correspondence $T$, and the modular generated by $T$ is denoted by $m(x)$.

3. Fréchet-derivatives and the Eigenvalue Problem. We will begin by the following

LEMMA 1. The functional of $\xi$ :

is differentiable and

$$
m(x+\xi y)
$$

$$
\frac{d}{d \xi} m(x+\xi y)=(T(x+\xi y), y)
$$

Proof. Although there are shorter proofs, we adopt here the one which uses a simple inequality between the modular and the conjugate modular. Putting $z=x+\xi y$ for any number $\varepsilon>0$, we have

$$
\begin{aligned}
m(x+(\xi+\varepsilon) y)-m(x+\xi y)- & (T(x+\xi y), \varepsilon y) \\
& =m(z+\varepsilon y)-m(z)-(T z, \varepsilon y) \\
& =m(z+\varepsilon y)-\{(T z, z)-\bar{m}(T z)\}-(T z, \varepsilon y) \\
& =m(z+\varepsilon y)+\bar{m}(T z)-(T z, z+\varepsilon y) \geqslant 0,
\end{aligned}
$$

and hence it follows that

$$
\lim _{\varepsilon \rightarrow 0} \frac{m(z+\varepsilon y)-m(z)}{\varepsilon} \geqslant(T z, y) .
$$

On the other hand,

$$
\begin{aligned}
m(z+\varepsilon y)-m(z) & =(T(z+\varepsilon y), z+\varepsilon y)-\bar{m}(T(z+\varepsilon y))-m(z) \\
= & (T(z+\varepsilon y), z)-m(z)+(T(z+\varepsilon y), \varepsilon y)-\bar{m}(T(z+\varepsilon y)) \\
\leqslant & (T(z+\varepsilon y), \varepsilon y)
\end{aligned}
$$

which implies that

$$
\varlimsup_{\varepsilon \rightarrow 0} \frac{m(z+\varepsilon y)-m(z)}{\varepsilon} \leqslant \varlimsup_{\varepsilon \rightarrow 0}(T(z+\varepsilon y), y)=(T z, y) .
$$

Therefore, the right-hand derivative of $m(x+\xi y)$ is $(T(x+\xi y), y)$ Analogously, we can prove that the left-hand derivative is also $(T(x+\xi y), y)$.

Remark. In this proof, we have used the following fact:

$$
\text { if } \lim _{\nu \rightarrow \infty}\left\|x_{\nu}-x\right\|=0 \text {, then } \lim _{\nu \rightarrow \infty}\left\|T x_{\nu}-T x\right\|=0 \text {. }
$$


This is true, because, since the norm is complete and continuous, the norm convergence is equivalent to the star-order-convergence (see [9], Theorems 33.4, 33.5), and $T$ is order-continuous.

Definition. A functional $f(x)$ on $R$ is said to be Fréchet-differentiable at $x$ if there exists an operator $F^{\prime} \in(R \rightarrow \bar{R})$ such that

and

$$
f(x+y)-f(x)=(F x, y)+\gamma(x, y)
$$

$$
\lim _{\|y\| \rightarrow 0} \frac{|\gamma(x, y)|}{\|y\|}=0 .
$$

The operator $F$ is called the gradient mapping of $f$, and it is, in general, non-linear. Sometimes we write $F x$ as $f^{\prime}(x)$. For various properties of the gradient mapping (see [5], [6], [10] and [11]).

Theorem 1. $m(x)$ is Fréchet-differentiable and

$$
m(x+y)-m(x)=(T x, y)+v(x, y)
$$

for the conjugately similar correspondence $T$.

Proof. For any $\varepsilon>0$ there exists $\delta=\delta(\varepsilon, x)>0$ such that $\|x-y\|<\delta$ implies $\|T x-T y\|<\varepsilon$. Therefore for those $\varepsilon, \delta$ we have

$$
\begin{aligned}
|m(x+y)-m(x)-(T x, y)| & =\left|\int_{0}^{1}\{(T(x+\xi y), y)-(T x, y)\} d \xi\right| \\
& \leqslant \int_{0}^{1}\|T(x+\xi y)-T x\| \cdot\|y\| \mid d \xi \leqslant \varepsilon\|y\|
\end{aligned}
$$

if $\|y\| \leqslant \delta$, because

$$
\|x+\xi y-x\| \leqslant \xi \cdot\|y\|<\delta \quad(0 \leqslant \xi \leqslant 1) .
$$

Therefore we have

$$
\varlimsup_{\|y\| \rightarrow 0} \frac{|\gamma(x, y)|}{\|y\|} \leqslant \varepsilon
$$

which is to be proved:

Definition. Let $F$ be an operator from $R$ into $\bar{R}$. A proper value $\lambda$ and a proper element $a \in R$ of $F$ are defined by

$$
F^{\prime} a=\lambda T a \text {. }
$$

In the case where $R=L_{p}(1<p<+\infty)$, this definition agrees with that of Citlanadze [4]. In particular, for $p=2$, we get the usual definition of the proper element in Hilbert spaces.
The following modification of a theorem of Ljusternik ([6], p. 239) is fundamental in our theory.

THEOREM 2. Let $S=\{x \in R: m(x)=1\}$ and let $N$ be a normal manifold of $R$. such that $S \cap N$ is not empty. Let $f(x)$ be a functional on $R$, which takes an extremum at $a \in S \cap N$, i.e.

$$
f(a) \leqslant f(x) \quad(\text { or } f(a) \geqslant f(x)) \quad \text { for every } x \in S \cap N .
$$

If $f(x)$ is Eréchet-differentiable at a, then

$$
f^{\prime}(a)[N]=\lambda T a,
$$

where $\lambda=\left(f^{\prime}(a), a\right) /\left(I^{\prime} a, a\right)$. Therefore, if $N=R$, then the element $a$ is a proper element of the gradient mapping $f^{\prime}(x)$ and the proper value, to which a belongs, is $f^{\prime}((a), a) /(T a, a)$.

Proof. Let us assume that $f(a) \leqslant f(x)(x \in S \cap N)$. Putting

$$
g(x)=f(x)-\lambda \cdot m(x),
$$

where $\lambda=\left(f^{\prime}(a), a\right) /(T a, a)$, we have

$$
g(a)=f(a)-\lambda m(a) \leqslant f(x)-\lambda m(x)=g(x) \quad(x \in S \cap N),
$$

which means that the function $g(x)$ also takes its minimum at $a$. Since $f(x)$ and $m(x)$ are Fréchet-differentiable, $g(x)$ is also Fréchet-differentiable and

$$
\left(g^{\prime}(a), a\right)=\left(f^{\prime}(a), a\right)-\lambda(T a, a)=0 .
$$

Now, for any $y \in R$ such that $a+\xi[N] y \neq 0$ for any $\xi$, the elements

$$
a(\xi)=a+\xi[N] y /\|a+\xi[N] y\| \| \quad(-\infty<\xi<+\infty)
$$

belong to $S \cap N$, and

$$
\begin{aligned}
g(a(\xi)) & =g(a)+\left(g^{\prime}(a), a(\xi)-a\right)+\gamma(a, a(\xi)-a) \\
& =g(a)+\left(g^{\prime}(a), a(\xi)\right)+\gamma(a, \dot{a}(\xi)-a) .
\end{aligned}
$$

Since $g(a) \leqslant g(a(\xi)) \quad(-\infty<\xi<+\infty)$, if $g(a(\xi))$ is right-hand differentiable at $\xi=0$, then the right-hand derivative must be non-negative.

We will prove that $g(a(\xi))$. is right-hand differentiable at $\xi=0$. For any $\varepsilon>0$, we have

$$
g(a(\varepsilon))-g(a)=\left(g^{\prime}(a), a(\varepsilon)\right)+\gamma(a, a(\varepsilon)-a),
$$

and

$$
\left(g^{\prime}(a), a(\varepsilon)\right)=\left(g^{\prime}(a), \frac{a+\varepsilon[N] y}{\|\mid a+\varepsilon[N] y\|}\right)=\frac{\varepsilon}{\|a+\varepsilon[N] y\|}\left(g^{\prime}(a),[N] y\right) .
$$


Therefore,

$$
\frac{g(a(\varepsilon))-g(a)}{\varepsilon}=\frac{1}{\|a+\varepsilon[N] y\|}\left(g^{\prime}(a),[N] y\right)+\frac{\gamma(a, a(\varepsilon)-a)}{\varepsilon} .
$$

On the other hand, since

$$
\begin{array}{r}
\frac{\| a(\varepsilon)-a] \|}{\varepsilon}=\frac{1}{\varepsilon}\left\|\left\{\left(\frac{1}{\|a+\varepsilon[N] y\|}-1\right) a+\frac{\varepsilon[N] y}{\|a+\varepsilon[N] y\|}\right\}\right\| \\
\leqslant \frac{1}{\varepsilon}\left|\frac{\|a+\varepsilon[N] y\|-1}{\|a+\varepsilon[N] y\|}\right|+\frac{\|[N] y\|}{\|a+\varepsilon[N] y\|},
\end{array}
$$

we have

$$
\begin{aligned}
& \varlimsup_{\varepsilon \rightarrow 0} \frac{|\gamma| a, a(\varepsilon)-a) \mid}{\varepsilon}=\varlimsup_{\varepsilon \rightarrow 0} \frac{\gamma \|(a, a(\varepsilon)-a) \mid}{\||| a(\varepsilon)-a \mid\|} \cdot \frac{\|a(\varepsilon)-a \mid\|}{\varepsilon} \\
& \leqslant \varlimsup_{\varepsilon \rightarrow 0} \frac{|\gamma(a, a(\varepsilon)-a)|}{\||a(\varepsilon)-a|\|} \cdot \mid \lim _{\varepsilon \rightarrow 0} \frac{|\|a+\varepsilon[N] y\|-\||\|a \mid\|}{\varepsilon}+\|[N .] y\| \|=0,
\end{aligned}
$$

because, by a result of S. Mazur [7],

$$
\lim _{\varepsilon \rightarrow 0} \frac{\||a+\varepsilon[N] y\||-|\| a \|}{\varepsilon}<+\infty
$$

and, by the definition of Fréchet-differentiation,

$$
\lim _{\varepsilon \rightarrow 0} \frac{|\gamma(a, a(\varepsilon)-a)|}{\|a(\varepsilon)-a\|||}=0 .
$$

Therefore we have

$$
\lim _{\varepsilon \rightarrow 0} \frac{g(a(\varepsilon))-g(a)}{\varepsilon}=\left(g^{\prime}(a),[N] y\right)=\left(g^{\prime}(a)[N], y\right) .
$$

Hence it follows that

$$
\left(g^{\prime}(a)[N], y\right) \geqslant 0 \quad(y \in R)
$$

which means that

$$
g^{\prime}(a)[N]=0
$$

Thus we obtain

$$
f^{\prime}(a)[N]=\lambda T a[N]=\lambda T a .
$$

Remark. In the definition of $S$ the condition that $m(x)=1$ is not essential. Namely, for

$$
S_{\lambda}=\{x \in R: m(x)=\lambda\}
$$

the modular

$$
m_{\lambda}(x)=\frac{1}{\lambda} m(x)
$$

enables us to get the same conclusion. By that $m_{\lambda}$ we can define a conjugately similar correspondence $T_{\lambda}$ which satisfies

Therefore

$$
T_{\lambda} x=\frac{1}{\lambda} T x .
$$

$$
f^{\prime}(a)[N]=\frac{\left(f^{\prime}(a), a\right)}{\left(T_{\lambda} a, a\right)} T_{\lambda} a=\frac{\left(f^{\prime}(a), a\right)}{(T a, a)} T a .
$$

For the properties of $m_{\lambda}$, we refer to [12].

LEMMA 2. Let us suppose that $f(x)(x \in R)$ satisfies the following two conditions:

i) $f(x)$ is weakly continuous;

ii) there exists a number $\alpha \geqslant 0$ such that $f(\xi x) \geqslant \xi^{a} f(x)$ for any number $\xi \geqslant 1$ and element $x \in R$.

Then, there exists an element $a \in R$ such that

$$
m(a)=1 \quad \text { and } \sup _{m(a)=1}|f(x)|=|f(a)| .
$$

Proof. The existence of such an element $a \in R$ that

$$
\sup _{m(x)=1}|f(x)|=|f(a)|
$$

follows from the facts that the unit sphere is weakly compact $\left({ }^{7}\right)$ and that $f(x)$ is weakly continuous. The norm of the element $a$ is not greater than one. If $m(a)<1$, then

$$
|f(a)| \geqslant\left|f\left(\frac{a}{\||a|\| \mid}\right)\right| \geqslant\left(\frac{1}{\||| a|| \mid}\right)^{a}|f(a)|,
$$

which shows that the element $a / \||| a|| \mid$ is our solution.

THEOREM. 3. Let us assume that:

i) $f(x)$ is Fréchet-differentiable;

ii) there exists a number $a \geqslant 0$ such that $f(\xi x) \geqslant \xi^{a} f(x)$ for any number $\xi \geqslant 1$ and element $x \in R$;

iii) $f(x)$ is positive: $f(x) \geqslant 0(x \in R)$;

iv) the gradient mapping $F$ of $f(x)$ is completely continuous. 
Then, there exists an element $a \in R$ such that $m(a)=1$ and

$$
F a=\frac{(F a, a)}{(T a, a)} T a
$$

namely, $a$ is a proper element of $F$ and the proper value to which a belongs is $(F a, a) /(T a, a)$.

Proof. By the assumptions ii), iii) and Lemma 2 , we can find $a \in R$ such that

$$
m(a)=1 \quad \text { and } \sup _{m(x)=1} f(x)=f(a)
$$

because the complete continuity of $F$ implies the weak continuity of $f(x)$ (see [10], Theorem 3.2). Therefore, by Theorem 2, we can complete this proof.

Before proceeding to the next theorem, we need the following

Definition. $A$ subset $A$ of $R$ is said to be complete if $x \perp A$ implies $x=0$. An element is said to be complete if it is complete as a one-point set.

THEOREM 4. We assume the conditions i)-iv) of Theorem 3. Moreover, we suppose that

v) there exists a complete element;

vi) $f(x) \leqslant f(|x|)$ for every $x \in R$.

Then there exists a sequence of projection operators $P_{v}$ in $\bar{R}$ such that

1) $P_{n} \downarrow_{p=1}^{\infty} 0$ and $P_{1}=\bar{I}$ (the identity in $\bar{R}$ );

2) there exist $a_{p} \geqslant 0$ and $\lambda_{p} \geqslant 0$ such that

$$
m\left(a_{v}\right)=1, \quad P_{v} F a_{v}=\lambda_{\nu} T a_{v} \quad \text { and } \quad P_{v}^{R} a_{\nu}=a_{v}\left({ }^{8}\right) ;
$$

3) the sequence $a_{v}(v=1,2, \ldots)$ is complete in $R$;

4) if $\lambda_{v} \neq 0(v=1,2, \ldots)$, then

$$
\inf _{\nu \geqslant 1} \lambda_{\nu}\left\|T a_{\nu}\right\|=0 \text {. }
$$

Proof. We start by the proper element $a$ of the preceeding theorem. By vi), we can take positive $a$. We denote it by $a_{1}$. When $a_{1}$ is not a complete element, then the non-empty set

$$
N_{a_{1}}=\left\{x \in R:\left(T a_{1},|x|\right)=0\right\}
$$

is normal and.

$$
\left[N_{a_{1}}\right]+\left[a_{1}\right]=I \quad(\text { the identity of } R) .
$$

(8) For the definition of $P_{y}^{R}$, see [9], p. 82 .
By the same method as in the proof of Lemma 2, we can find $a_{2} \in R$ such that $m\left(a_{2}\right)=1, a_{2} \in\left[N a_{1}\right]$ and

$$
\sup _{m(x)=1, x_{6} N a_{1}} f(x)=f\left(a_{2}\right)
$$

Then, by Theorem 3 , we have

$$
F a_{2}\left[N a_{1}\right]=\lambda_{2} T a_{2},
$$

where

$$
\lambda_{2}=\left(F a_{2}, a_{2}\right) /\left(T a_{2}, a_{2}\right)=\left(F a_{2}, a_{2}\right) /\left\|T a_{2}\right\| .
$$

Suppose that we could find an orthogonal $a_{\nu}>0(v=1,2, \ldots, \mu)$ such that

$$
m\left(a_{v}\right)=1, \quad a_{v} \in N a_{1} \cap N a_{2} \cap \ldots \cap N a_{n-1}
$$

and

$$
F a_{v}\left[N a_{1}\right]\left[N a_{2}\right] \ldots\left[N_{v-1}\right]=\lambda_{v} T a_{v} \quad \text { where } \quad \lambda_{v}=\left(F a_{v}, a_{v}\right) /\left\|T a_{v}\right\| .
$$

If $\left\{a_{v}(v=1,2, \ldots, \mu)\right\}$ is not complete, we can continue this process. Since $R$ is superuniversally continuous, the set of such elements must be at most countable. Therefore, 3 ) is true.

Now, since

$$
\left[N a_{v}\right]=I-\left[a_{v}\right]=I-\left[T a_{p}\right]^{R}
$$

we have

$$
\begin{aligned}
F a_{v}\left[N a_{1}\right]\left[N a_{2}\right] \ldots\left[N a_{v-1}\right] & =F a_{v}\left(I-\left[T a_{1}\right]^{R}\right) \ldots\left(I-\left[T a_{v-1}\right]^{R}\right) \\
& =\left(\bar{I}-\left[T a_{1}\right]\right) \ldots\left(\bar{I}-\left[T a_{v-1}\right]\right) F a_{p} .
\end{aligned}
$$

Putting $P_{1}=\bar{I}$ and

$$
P_{v}=\left(\bar{I}-\left[T a_{1}\right]\right) \ldots\left(\bar{I}-\left[T a_{p-1}\right]\right) \quad(v=2,3, \ldots),
$$

we have

$$
P_{,} F a_{\nu}=\lambda_{,} T a_{,} \quad(\nu=1,2, \ldots)
$$

and

$$
P_{v}^{R} a_{v}=\left[N a_{1}\right] \ldots\left[N a_{v-1}\right] a_{v}=a_{v} \quad(v=2,3, \ldots) .
$$

Thus, we could prove 2 ).
It is evident that $P_{1} \geqslant P_{2} \geqslant \ldots$ We will prove that $\bigcap_{y=1}^{\infty} P_{v}=0$ (the projection operator defined by $0 \cdot \bar{x}=0)$. Since

$$
P_{\nu}^{R}=\left[N a_{1}\right] \ldots\left[N a_{p-1}\right]
$$


we have only to prove that

$$
\bigcap_{v=2}^{\infty}\left[N a_{1}\right] \ldots\left[N a_{y-1}\right]=0 .
$$

Suppose, on the contrary, that

$$
\bigcap_{v=2}^{\infty}\left[N a_{1}\right] \ldots\left[N a_{v-1}\right] \neq 0 .
$$

Then there exists a projector $[p] \neq 0$ such that

$$
[p] \leqslant\left[N a_{1}\right] \ldots\left[N a_{\nu-1}\right] \quad(\nu=2,3, \ldots),
$$

which shows that

$$
[p]\left[a_{v}\right]=0 \quad(v=1,2, \ldots) .
$$

This contradicts the fact that $\left\{a_{v}(\nu=1,2, \ldots)\right\}$ is complete.

Finally, we will prove that 4 ) is true. If

$$
\inf _{v \geqslant 1} \lambda_{v}\left\|T a_{v}\right\| \neq 0
$$

we can take a subsequence (we denote it also by $\lambda_{\nu}\left\|T a_{\nu}\right\|$ ) such that $\lambda_{\nu}\left\|T a_{v}\right\|>\varepsilon(\nu=1,2, \ldots)$ for some $\varepsilon>0$. Then the elements $a_{v} / \lambda_{\nu}\left\|T a_{\nu}\right\|$ $(v=1,2, \ldots)$ are norm-bounded. Therefore, by iv), the set

$$
\left\{\frac{F a_{\nu}}{\lambda_{v}\left\|T a_{v}\right\|} \quad(v=1,2, \ldots)\right\}
$$

is compact, and hence it follows that

$$
\left\{\frac{P_{v} F a_{v}}{\lambda_{\nu},\left\|T a_{v}\right\|}=\frac{T a_{v}}{\left\|T a_{v}\right\|} \quad(v=1,2, \ldots)\right\}
$$

is compact. But this is impossible, because, as $a_{v}(v=1,2, \ldots)$ are mutually orthogonal, we have

$$
\begin{aligned}
\bar{m}\left(\frac{T a_{v}}{\left\|T a_{\nu}\right\|}-\frac{T a_{\mu}}{\left\|T a_{\mu}\right\|}\right) & =\bar{m}\left(\frac{T a_{v}}{\left\|T a_{\mu}\right\|}+\frac{T a_{\mu}}{\left\|T a_{\mu}\right\|}\right) \\
& \geqslant \bar{m}\left(\frac{T a_{\nu}}{2\|\| T a_{\nu} \|}\right)+\bar{m}\left(\frac{T a_{\nu}}{2\|\| T a_{\mu} \|}\right) \geqslant 2 \inf _{\bar{m}(\bar{x})=1} \bar{m}\left(\frac{1}{2} \bar{x}\right)>0,
\end{aligned}
$$

since $\bar{R}$ is uniformly simple ([13], Lemma 2.1). Thus 4) is established.

Remark. It is possible that, for an $\alpha$, there exist infinite $x_{\lambda} \geqslant 0$ $(\lambda \in \Lambda)$ such that

$$
F_{x_{\lambda}}=\alpha T x_{\lambda} \quad \text { and } \quad m\left(x_{\lambda}\right)=1
$$

But the set $\left\{x_{\lambda}(\lambda \in \Lambda)\right\}$ contains only a finite. number of mutually orthogonal elements because of the complete continuity of $F$. It is evident that mutually orthogonal elements are linearly independent. But the inverse is not always true.

4. Symmetric operators. In this section we will introduce the notion of symmetric operators on Banach spaces and apply the results of the preceeding section.

Definition. An operator $F \epsilon(R \rightarrow \bar{R})\left({ }^{9}\right)$ is said to be symmetric if $(F x, y)=(F y, x)(x, y \in R)$.

Obviously the symmetric operator is additive and homogeneous. The conjugately similar correspondence $T$ is symmetric if and only if the space $R$ is an abstract $L_{2}$-space (").

For a symmetric operator $F$, we put

$$
f_{F}(x)=\frac{1}{2}(F x, x) \text {. }
$$

Then $f_{F}(x)$ is homogeneous of order 2, that is, a quadratic form. Moreover, we have

LEMMA 3. If $F$ is continuous and symmetric, $f_{F}(x)$ is Fréchetdifferentiable and $f_{F}^{\prime}(x)=F x$.

Proof. By (*), we have

$$
\begin{aligned}
f_{F}(x+y)-f_{F}(x) & =\frac{1}{2}\{(F(x+y), x+y)-(F x, x)\} \\
& =(F x, y)+\frac{1}{2}(F y, y),
\end{aligned}
$$

and $|(F y, y)| \leqslant\|F y\| \cdot\|y\|$. Therefore

$$
\lim _{\|y\| \rightarrow 0} \frac{|(F y, y)|}{\|y\|}=0,
$$

because $F$ is continuous. Thus we have

$$
\left(f^{\prime}(x), y\right)=(F x, y) \quad(y \in R) .
$$

() We will denote by $(R \rightarrow \widetilde{R})$ the set of all operators from $R$ into $\bar{R}$.

(20) If $F$ is symmetric, it is homogeneous. Therefore

$$
m(\xi x)=\int_{0}^{\xi}(T \eta x, x) d \eta=\frac{\xi^{2}}{2}(T x, x),
$$

which means that $m(\xi x)=\xi^{2} m(x)$. Hence it follows that $\|x+y\|^{2}=\|x\|^{2}+\|y\|^{2}$ if $x \cap y=0$. See [3]. 
Remark. If a function $f(x)$ is Fréchet-differentiable and its gradient mapping $F$ is symmetric, then

$$
f(x)=\frac{1}{2}(F x, x)+f(0) .
$$

In fact,

$$
\begin{aligned}
f(x)-f(0) & =\int_{0}^{1}(F(\xi x), x) d \xi \\
& =\int_{0}^{1} \xi(F x, x) d \xi=\frac{1}{2}(F x, x) .
\end{aligned}
$$

Definition. An operator $F \epsilon(R \rightarrow \bar{R})$ is said to be positive definite, if $(F x, x) \geqslant 0(x \in R) . F$ is said to be positive if $x \geqslant 0$ implies $F x \geqslant 0$. Obviously, these two notions are independent.

Lemma 3. If $F$ is positive, then $f_{F^{\prime}}(x) \leqslant f_{F}(|x|)$.

Proof.

$$
\begin{aligned}
2 f_{F}(x) & =(F x, x) \\
& =\left(F\left(x^{+}-x^{-}\right), x^{+}-x^{-}\right) \\
& =\left(F x^{+}, x^{+}\right)-2\left(F x^{+}, x^{-}\right)+\left(F x^{-}, x^{-}\right) \\
& \leqslant\left(F x^{+}, x^{+}\right)+2\left(F x^{+}, x^{-}\right)+\left(F x^{-}, x^{-}\right) \\
& \left.=(F|x|,|x|)=2 f_{F}(|x|){ }^{11}\right) .
\end{aligned}
$$

Thus, we can easily see that the following theorem is true:

THEOREM 5. If $F$ is symmetric, completely continuous, positive and positive definite, then the functional $f_{F}(x)$ satisfies the conditions i)-iv) and vi) of Theorem 3 and Theorem 4 . Therefore we can apply Theorem 4. In this case we can replace 4 ) of Theorem 4 by

$\left.4^{\prime}\right)$ if $\lambda_{p}>0$, then $\lambda_{1}\left\|T a_{1}\right\| \geqslant \lambda_{2}\left\|T a_{2}\right\| \geqslant \ldots$ and

$$
\lim _{\nu \rightarrow \infty} \lambda_{\nu}\left\|T a_{\nu}\right\|=0 \text {. }
$$

In fact, $\lambda_{p}\left\|T a_{\nu}\right\|=\left(F a_{p}, a_{v}\right)=2 f_{F i}\left(a_{\nu}\right)$.

Remark. If a symmetric operator $F$ is positive definite, we can easily prove the following equality:

$$
\left\|F^{\prime}\right\|=\sup _{\|x \mid\|=1}\|F x\|=\sup _{m(x)=1}(F x, x) .
$$

(11) $|x|=x^{+}+x^{-}, x^{+}=x \cup 0$ and $x^{-}=(-x)^{+}$.
5. An integral operator on modulared function spaces. For $\xi>0$ and $0 \leqslant t \leqslant 1$, we consider a finite function $\varphi(\xi, t)$, which is assumed to be strictly increasing with respect to $\xi>0$, measurable with respect to $t$ and $\varphi(0, t)=0$ for almost all $t$. Let the inverse function of $\varphi(\xi, t)$ as a function of $\xi$ be $\psi(\xi, t)$, which is also a finite, strictly increasing function of $\xi>0$ and measurable with respect to $t$.

We define Young's functions as follows:

$$
\Phi(\xi, t)=\int_{0}^{\xi} \varphi(\eta, t) d \eta \quad \text { and } \quad \Psi(\xi, t)=\int_{0}^{\xi} \psi(\eta, t) d \eta .
$$

Then it is easy to see that

$$
\xi \eta \leqslant \Phi(\xi, t)+\Psi(\eta, t)
$$

and

$$
\alpha \beta=\Phi(\alpha, t)+\Psi(\beta, t) \quad \text { if } \quad \beta=\varphi(\alpha, t) \text { or } \alpha=\psi(\beta, t) .
$$

Let $L_{\phi}$ be the totality of all measurable functions $x(t) \quad(0 \leqslant t \leqslant 1)$ such that

$$
m(\xi x)=\int_{0}^{1} \Phi(\xi|x(t)|, t) d t<+\infty \quad \text { for some } \quad \xi>0 .
$$

Then the functional $m(x)$ satisfies the "modular conditions" of [9], p. 153. This space $L_{\Phi}$ is reflexive and its conjugate space is $L_{\psi}$. Every element $\bar{x} \epsilon \bar{L}_{\phi}$ takes the form of

$$
\bar{x}(x)=\int_{0}^{1} x(t) \bar{x}(t) d t \quad\left(x(t) \epsilon L_{\varphi}\right)
$$

for some $\bar{x}(t) \epsilon L_{\Psi}$.

Throughout this section we assume that $m(x)<+\infty$ for every $x(t) \in L_{\Phi}$.

LEMMA 4. The transformation

$$
T x(t)=\varphi\left(x^{+}(t), t\right)-\varphi\left(x^{-}(t), t\right)
$$

is a conjugately similar correspondence between $L_{\Phi}$ and $L_{\Psi}$. Then

Proof. At first, we prove that $T \epsilon\left(L_{\Phi} \rightarrow L_{Y r}\right)$. Let $0 \leqslant x(t) \in L_{\Phi}$.

$$
\int_{0}^{1} x(t) \cdot \varphi(x(t), t) d t=\lim _{\varepsilon \rightarrow 0} \frac{m((1+\varepsilon) x)-m(x)}{\varepsilon}<+\infty .
$$


Therefore we have

$$
\int_{0}^{1} \Psi(\varphi(x(t), t)) d t=\int_{0}^{1} x(t) \cdot \varphi(x(t), t) d t-\int_{0}^{1} \Phi(x(t), t) d t<+\infty
$$

which means that

$$
T x(t) \in L_{\Psi} \quad \text { if } \quad 0 \leqslant x(t) \epsilon L_{\Phi} .
$$

For arbitrary $x(t) \epsilon L_{\Phi}$ we have

$$
T x(t)=T x^{+}(t)-T x^{-}(t) \epsilon L_{\Psi} .
$$

Next we prove that

$$
T x=T y \quad \text { implies } \quad x=y .
$$

We can assume that $x(t), y(t)$ are positive. If $x(t) \neq y(t)$, then, for example,

$$
x(t)>y(t)
$$

on a set of positive measure. Therefore, we can find $\alpha>\beta>0$ such that the set

$$
\{t: x(t)>\alpha>\beta>y(t)\}
$$

is of positive measure. Since $\varphi(\xi, t)$ is strictly increasing, we have $\varphi(x(t), t) \neq \varphi(y(t), t)$.

To show that $T$ is one-to-one, we have only to prove that for any $0 \leqslant y \in L_{\Psi}$ there exists $0 \leqslant x \in L_{\Phi}$ such that $T x=y$. But this is obvious, because, for that $y(t) \in L_{\Psi}$. we have

$$
x(t)=\psi(y(t), t) \epsilon L_{\Phi} \quad \text { and } \quad \varphi(x(t), t)=y(t) .
$$

We find no difficulty in proving the following conditions: (1) $T(-x)$ $=-T x ;(2) x \leqslant y$ if and only if $T x \leqslant T y ;(3)(T x, x)=0, x \geqslant 0$ implies $x=0$.

Thus the proof is established.

Next we will consider the following integral operator:

$$
F x=y(s)=\int_{0}^{1} K(s, t) x(t) d t .
$$

Using a method of A. C. Zaanen [14], we can prove the following TheOREM 6. If

$$
\int_{0}^{1} \Psi\left[\int_{0}^{1} \Psi(|K(s, t)|, t) d t, s\right] d s<+\infty,
$$

then the operator $(* *)$ is completely continuous as an operator from $L_{\phi}$ into $L_{\Psi}$.

Proof. At first, we prove that $F \epsilon\left(L_{\Phi} \rightarrow L_{\Psi}\right)$. For $F x=y$, we have

$$
\begin{aligned}
\int_{0}^{1} \Psi\left(\frac{1}{2}|y(s)|, s\right) d s= & \int_{0}^{1} \Psi\left[\frac{1}{2}\left|\int_{0}^{1} K(s, t) x(t) d t\right|, s\right] d s \\
\leqslant & \int_{0}^{1} \Psi\left[\frac{1}{2} \int_{0}^{1} \Psi(|K(s, t)|, t) d t+\frac{1}{2} \int_{0}^{1} \Phi(|x(t)|, t) d t, s\right] d s \\
\leqslant & \frac{1}{2} \int_{0}^{1} \Psi\left[\int_{0}^{1} \Psi(|K(s, t)|, t) d t, s\right] d s \\
& +\frac{1}{2} \int_{0}^{1} \Psi\left[\int_{0}^{1} \Phi(|x(t)|, t) d t, s\right] d s<+\infty
\end{aligned}
$$

which shows that $F x \in L_{\Psi}$.

To prove that $F$ is completely continuous, we note that in this case the norm convergence and the convergence by modular $\left(^{12}\right)$ coincide ([13], Lemma 2.1).

Let a set $A \subset L_{\Phi}$ be bounded, namely,

$$
\|x\| \leqslant \gamma \quad(x \in A)
$$

for some $\gamma>0$. This is equivalent to

$$
m\left(\frac{1}{\gamma} x\right) \leqslant 1 \quad(x \in A) .
$$
that

Since $A$ is weakly compact, there exist $x_{v}(v=0,1,2, \ldots)$ such

$$
\lim _{y \rightarrow \infty} x_{p}=x_{0} \quad \text { weakly and } \quad m\left(2 x_{v}\right) \leqslant 1,
$$

where $2 \gamma x_{\nu} \in A$. Therefore, for every $y(t) \epsilon L_{\Psi}$, we have

$$
\lim _{\nu \rightarrow \infty} \int_{0}^{1} x_{\nu}(t) y(t) d t=\int_{0}^{1} x_{0}(t) y(t) d t .
$$

The assumption on $K(s, t)$ shows that $K(s, t) \epsilon L_{\Psi}$ as a function of $t$ for almost all $s$. Hence it follows that for

$$
y_{v}(s)=\int_{0}^{1} K(s, t) x_{v}(t) d t
$$

(12) A sequence $x_{y}$ is said to be convergentby modular to $x_{0}$ if $\lim _{y \rightarrow \infty} m\left(x_{y}-x_{0}\right)=0$. 
we have

$$
\lim _{v \rightarrow \infty}\left|y_{v}(s)-y_{0}(s)\right|=0 \quad \text { almost everywhere, }
$$

which implies

$$
\lim _{v \rightarrow \infty} \Psi\left(\left|y_{v}(s)-y_{0}(s)\right|, s\right)=0 \quad \text { almost everywhere. }
$$

On the other hand, since

$$
\begin{aligned}
\left|y_{v}(s)-y_{0}(s)\right| & \leqslant \int_{0}^{1}|K(s, t)| \cdot\left|x_{v}(t)-x_{0}(t)\right| d t \\
& \leqslant \int_{0}^{1} \Psi(|K(s, t)|, t) d t+\int_{0}^{1} \Phi\left(\left|x(t)-x_{0}(t)\right|, t\right) d t \\
& \leqslant \int_{0}^{1} \Psi(|K(s, t)|, t) d t+1
\end{aligned}
$$

we have

$$
\Psi\left(\left|y_{v}(s)-y_{0}(s)\right|, s\right) \leqslant \Psi\left[\int_{0}^{1} \Psi(|K(s, t)|, t) d t+1, s\right],
$$

and the right-hand function of $s$ is integrable. Therefore, by a theorem of Lebesgue, we have

$$
\lim _{p \rightarrow \infty} \int_{0}^{1} \Psi\left(\left|y_{v}(s)-y_{0}(s)\right|, s\right) d s=0 .
$$

This means that $F x^{\prime}$, converges to $F x_{0}$ by the modular convergence. Therefore,

$$
\lim _{v \rightarrow \infty}\left\|F x_{p}-F x_{0}\right\|=0
$$

by the norms defined by the modular. Since $2 \gamma x_{\nu} \in A$ and $F$ is linear, $F(A)$ is compact.

Remark. If $\Phi(\xi, t)=\Phi(\xi)$, i. e. if $\Phi(\xi, t)$ is a function of only $\xi>0, L_{\Phi}$ is an Orlicz space. If $\Phi(\xi, t)=\xi^{p(t)}$ for a measurable function $p(t) \geqslant 1, L_{\Phi}$ is $L_{p(t)}$. But in those cases we cannot always obtain the exact forms of their norms that make them Banach spaces. It is easy to see that in the case where

$$
\Phi(\xi, t)=\xi^{p} \quad \text { for } \quad p>1,
$$

i. e. in the case of $L_{p}(p>1)$, the condition on $K(s, t)$ of the above theorem can be replaced by a better one:

$$
\int_{0}^{1} \int_{0}^{1}|K(s, t)|^{p /(p-1)} d s d t<+\infty
$$

By Theorem 4 and 6, we can ensure the existence of a positive proper element of the operator (**). Namely,

THEOREM 7. Let us suppose that the operator (**) satisfies the following conditions:

i) $\int_{0}^{1} \Psi\left[\Psi \int_{0}^{1}(|K(s, t)| t) d t, s\right] d s<+\infty$;

ii) $K(s, t) \geqslant 0$ for every $s, t$;

iii) $K(s, t)=K(t, s)$.

Then, there exist a positive number $a$ and a function $a(t) \in L_{\Phi}$ such that

$$
\int_{0}^{1} K(s, t) a(t) d t=\lambda \varphi(a(s), s) .
$$

If, moreover, $F$ is positive definite, then there exists a sequence of measurable sets $E_{v}(v=1,2, \ldots)$ such that:

1) $[0,1]=E_{1} \supset E_{2} \supset \ldots$ and $\lim \left|E_{p}\right|=0$;

2) there exist $a_{v}(t) \geqslant 0$ and $\lambda_{\nu} \geqslant 0$ such that

$$
\int_{0}^{1} \Phi\left(a_{p}(t), t\right) d t=1 \quad \text { and } \quad \int_{E_{v}} K(s, t) a_{\nu}(t) d t=\lambda_{\nu} \varphi\left(a_{v}(s), s\right) ;
$$

3) if $a_{v}(t) \cdot x(t)=0(v=1,2, \ldots)$ and $x(t) \geqslant 0$, then $x=0$;

4) if $\lambda_{v}>0(\nu=1,2, \ldots)$, then $\lim \lambda_{\nu}=0$.

\section{References}

[1] I. A memiya, A generalization of Riesz-Fisher's theorem, Jour. Math. Soc. Japan 5 (1953), p. 353-354.

[2] G. Birkhoff, Lattice Theory, American Math. Soc. 1949.

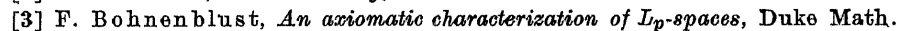
Journal 6 (1940), p. $627-640$

[4] E.S. Citlanadze, The method of orthogonal trajectories for non-linear operators of variational type in the space $L_{p}$, Acad. Nauk Gruzin. SSR, Trudy Tbiliss. Mat. Inst. Razmadze 20 (1954), p. 245-278; American Math. Soc. Transactions, Series 2, Vol. 5, p. 305-333.

[5] M. A. Krasnoseliskii and J. B. Rutickii, Oonvex functions and Orliaz spaces (Russian), 1958.

[6] L. A. Ljusternik and W. I. Sobolev, Flemente der Funktionalanalysis, 1955.

[7] S. Mazur, Über konvexe Mengen lin linearen normierten Räumen, Studia Math. 4 (1933), p. $70-84$.

[8] E. J. MeShane, Integration, Princeton 1944

[9] H. Nakano, Modulared semi-ordered linear spaces, Tokyo 1950. 
[10] E. Rothe, Gradient mappings and extrema in Banach spaces, Duke Math. Jour. 15 (1948), p. 421 - 431

[11] M. M. Vainberg, Some questions of differential calculus in linear spaces, Uspohi Mat. Nauk, N.S. 7 (1952), p. $55-102$ (Russian).

[12] S. Yamamuro, On conjugate spaces of Nakano spaces, Transactions Amer. Math. Soc. 90 (1959), p. $291-311$.

[13] - Monotone completeness of normed semi-ordered linear spaces, Pacifio Jour. Math. 7 (1957), p. 1715-1725.

[14] A. C. Záanen, On a certain class of Banach spaces, Annals of Math. 47 (1946), p. $654-666$.

\section{HOKKAIDO UNIVERSITY}

SAPPORO, JAPAN

Regu par la Rédaction le 18.3.195
Remarks to the paper of $\mathrm{L}$. Kubik "The limiting distributions of cumulative sums of independent two-valued random variables" (Studia Mathematica 18(1959), p. 295 - 309)

In the definition of class $\mathscr{C}$ it is necessary to assume additionally that the limit

$$
\lim _{n \rightarrow \infty} z_{n} / \sqrt{\sum_{k=1}^{n} D^{2}\left(X_{k}\right)}
$$

exists and in the definition of class $\mathcal{X}$ (and in the proof of theorem) it is necessary to assume that $A=-B, \nu+\mu=1$. In the proof of theorem, $\mathfrak{P}(n)$ and $\mathfrak{Q}(n)$ should be defined as $\mathfrak{P}(n)=\mathfrak{P} \cap \mathfrak{N}(n)$, $\mathfrak{Q}(n)=\mathfrak{Q} \cap \mathfrak{N}(n)$ where $\mathfrak{P}$ (respectively $\mathfrak{Q}$ ) denotes the set of positive integers $k$ such that $\min \left(p_{k}, q_{k}\right)=p_{k}$ (respectively $\left.\min \left(p_{k}, q_{k}\right)<p_{k}\right)$.

The theorem on page 296 should be formulated as follows:

The class $\mathcal{R}$ coincides with the olass of all distributions which are of the same type as any element of $\mathcal{K}$.

The elements of $\mathcal{R}$ which do not belong to $\mathcal{X}$ can be obtained by replacing $B_{n}=\sqrt{\sum_{k=1}^{n} D^{2}\left(X_{k}\right)}$ by $B_{n}^{\prime}=B_{n} / \sigma$. 